\title{
Alexitimia, resiliencia y creencias paranormales en personas mayores en un centro institucionalizado
}

\author{
Inmaculada Méndez ${ }^{1}$, Julia García-Sevilla ${ }^{1}$, Juan P. Martínez ${ }^{1}$, \\ Ana $\mathrm{M}^{\mathrm{a}}$ Bermúdez ${ }^{1}$, Pilar Pérez ${ }^{1}$ e Isabel García-Munuera ${ }^{2}$ \\ ${ }^{1}$ Universidad de Murcia (España); ${ }^{2}$ Universidad de Granada (España)
}

La alexitimia hace referencia a la dificultad para comprender e identificar los propios sentimientos y los de los demás. El proceso de adaptación a un centro residencial es una de las situaciones estresantes a las que puede enfrentarse la persona mayor. Para poder sobreponerse ante dichas situaciones es fundamental la capacidad de resiliencia e incluso existen personas que buscan apoyo en la religiosidad o en las creencias en lo paranormal. En este trabajo se estudió la relación entre alexitimia, resiliencia y creencias paranormales en un grupo de 35 personas mayores (21 mujeres) con edades entre 66 y 95 años de un centro institucionalizado. Se administraron la escala TAS-20 para la evaluación de la alexitimia, la Escala de Resiliencia CD-RISC y la Escala Mejorada de Creencias Paranormales. Entre los principales resultados cabe destacar que los sujetos con mayores Dificultades para identificar sentimientos son los que presentaban un menor Control personal; los sujetos con mayores Dificultades para identificar sentimientos son los que menor creencia poseían de las Formas de vida extraordinaria y los sujetos con mayor capacidad de Control Personal son los que mayor creencia tenían de las Formas de vida extraordinaria. Se discuten las aplicaciones que los profesionales pueden poner en marcha en centros institucionalizados de personas mayores.

Palabras clave: Alexitimia, resiliencia, creencias paranormales, personas mayores.

Alexithymia, resilience and paranormal beliefs in an elderly institutionalized center. Alexithymia refers to the difficulty to understand and identify feelings and those of others. The process of adapting a residential facility is one of the stressful situations older people may face. In order to overcome this situation, resilience is critical and even there are people who seek support in religion or paranormal beliefs. In this paper, we studied the relationship between alexithymia, resilience and paranormal beliefs in a group of 35 seniors (21 women) aged between 66 and 95 years old in an institutionalized center. The instruments used were the TAS-20 Scale in order to assess alexithymia, the CD-RISC for evaluating resilience and the Enhanced Paranormal Beliefs Scale. Among main findings, it must be emphasized that subjects with greater difficulty identifying feelings are those with less personal control and less belief about extraordinary life forms; subjects with greater personal control had higher belief about those extraordinary life forms. Professional applications that can be launched in elderly institutionalized centers are discussed.

Keywords: Alexithymia, resilience, paranormal beliefs, elderly.

Correspondencia: Inmaculada Méndez. Facultad de Psicología. Departamento de Psicología Evolutiva y de la Educación. Universidad de Murcia. Campus de Espinardo. C.P. 30100. Murcia (España). E-mail: inmamendez@um.es 
El concepto de alexitimia alude a la dificultad que presenta un individuo para poder comprender los sentimientos de los otros. Del mismo modo hace referencia a un estilo cognitivo que se caracteriza por: la dificultad para poder expresar estados afectivos y distinguirlos de otras sensaciones corporales, la falta de capacidad para la introspección, pueden caer en el conformismo social por no confiar en su criterio para discernir el valor afectivo de la situación y la presencia de conductas de acción frente a situaciones de conflicto (Nemiah, 1977; Martínez-Sánchez, Ato-García, y Ortiz-Soria, 2003; Sivak y Wiater, 1997). Por lo tanto, la alexitimia es considerada por algunos autores como una dislexia afectiva, dado que el sujeto no es capaz de discriminar matices afectivos (Bodni, 1991). Estudios previos consideran que se trata de un concepto que ha de ser entendido situándolo en un extremo del continuum que describe la comunicación de tipo emocional (Lolas, 1978). Las investigaciones realizadas la han asociado con dolencias de tipo psicosomático, enfermedades físicas y psicológicas, como por ejemplo, García-Sevilla et al. (2014), quienes destacaron que aquellos ancianos con un patrón alexitímico presentaban sintomatología depresiva.

Unido a lo anterior, es destacable que los centros residenciales son el recurso más conocido, utilizado y que posee más beneficiarios para las personas mayores en nuestro país (Pérez-Fuentes, Molero, y Gázquez, 2011) debido al aumento de la dependencia (Gázquez, Pérez-Fuentes, Mercader, y Molero, 2011). El proceso de adaptación a un centro residencial es una de las situaciones a las que puede enfrentarse la persona en la tercera edad al igual que otras situaciones estresantes como la muerte de seres queridos, la jubilación (González-Arratia y Valdez, 2013), cambios económicos e incluso restricciones en la participación social (Cárdenas-Jiménez y López-Díaz, 2011). Para poder sobreponerse ante dichas situaciones es fundamental la capacidad de resiliencia. Se ha conceptualizado como la combinación o interacción dinámica entre los procesos de riesgo y los de protección individual y del ambiente familiar, social y cultural del sujeto con la finalidad de superar los efectos de los eventos adversos de la vida (González-Arratia y Valdez, 2013). A veces para sobreponerse a dichas situaciones existen personas que buscan apoyo en la religiosidad, sobre todo en los ancianos, en lo referente a las creencias y asistencia a los servicios religiosos (Cárdenas y López, 2010). En este sentido, la religiosidad tiene dos orientaciones: a nivel intrínseco (intrapersonal) y a nivel extrínseco (interpersonal). Dichas orientaciones se han asociado con la salud en los ancianos: disminuyen la mortalidad por problemas cardiacos, disminuyen el estrés y la depresión; aumentan el nivel de satisfacción de vida y por ende el bienestar. Del mismo modo, disminuyen la sintomatología ansiosa y depresiva ante la muerte (Parra y Corbetta, 2013). Por lo tanto, las creencias religiosas, según Reyes- Ortiz (1998), provee a la persona de esperanza y autoestima, proporcionando un soporte socioemocional y promoviendo mecanismos psicológicos adaptativos ante el proceso de envejecimiento, la enfermedad y el sufrimiento. Asimismo, poseer un sistema de creencias/experiencias 
paranormales (interpretaciones más placenteras de la realidad) puede actuar como mecanismo de protección, favoreciendo que los individuos acepten y expliquen sus experiencias, mientras que aquellos que carecen de él pueden ser más vulnerables a la perturbación emocional que desencadene la experiencia vivida. Por lo tanto, el hecho de poseer ese sistema de creencias probablemente puede dar lugar a experiencias paranormales adaptativas que han sido asociadas a una reducción del nivel de depresión, ansiedad y anhedonia (Parra y Corbetta, 2013).

Tras lo mencionado, este trabajo tiene el objetivo de estudiar la relación existente entre la alexitimia, la resiliencia y las creencias paranormales en personas mayores en un centro institucionalizado.

\section{MÉTODO}

\section{Participantes}

El tipo de muestreo llevado a cabo en el estudio fue de tipo intencional. Para ello como criterios de inclusión se tuvieron en cuenta los siguientes: ser residentes en la institución seleccionada y presentar según la Escala de Deterioro Global (GDS- Global Deterioration Scale-) de Reisberg, Ferris, León, y Crook (1982), un nivel máximo de GDS 5, es decir, un deterioro cognitivo moderadamente grave o demencia moderada, de forma que fuese posible cumplimentar los instrumentos de evaluación. Los criterios de exclusión fueron: poseer un nivel de deterioro global superior al GDS 6 o 7 compatibles con demencia moderadamente grave o grave lo que imposibilitaría responder los instrumentos utilizados.

Los sujetos del estudio fueron 35 personas mayores de los 79 del total que residían en un centro institucionalizado de Murcia. En lo referente al sexo, el $60 \%$ eran mujeres $(N=21)$ y con edades comprendidas entre los 66 y los 95 años (véase gráfico 1). En cuanto al estado civil hubo $17.1 \%$ de solteros, $20 \%$ de casados, $54.3 \%$ de viudos y el $8.3 \%$ de separados/ divorciados.

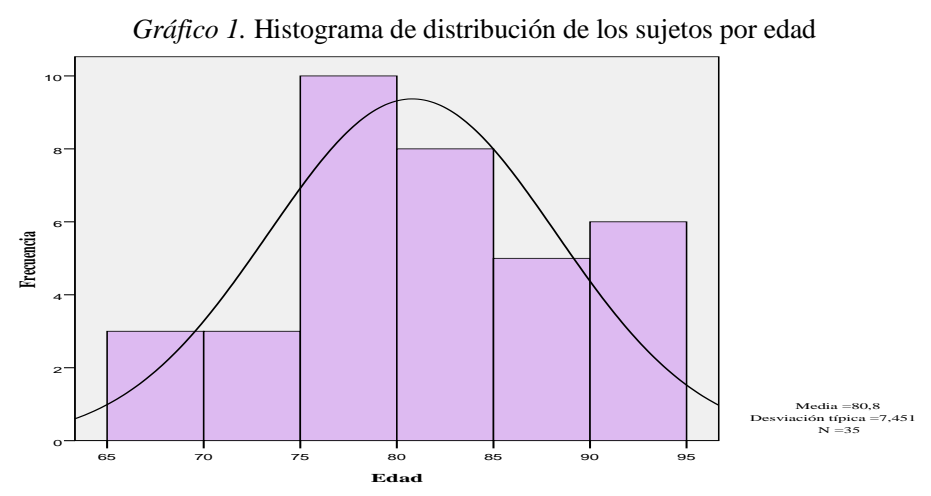




\section{Instrumentos}

A continuación se describen brevemente los instrumentos utilizados:

Para evaluar la alexitimia se utilizó la escala TAS-20. Se trata de la escala abreviada de Alexitimia de Toronto (TAS-20- Twenty-Item Toronto Alexithymia Scale-) de Bagby, Parker, y Taylor (1994) que fue adaptada a la población española por Martínez-Sánchez (1996). La escala consta de 20 ítems con escala tipo Likert con un rango de respuesta que oscila entre: 1 nada a 5 mucho. Se compone de tres subescalas: a) dificultad para identificar los sentimientos y distinguirlos de las sensaciones fisiológicas que acompañan a la activación emocional (7 ítems); b) dificultad para describir y expresar los sentimientos (5 ítems) y c) pensamiento orientado hacia lo externo ( 8 ítems). La suma de las tres subescalas conforman la escala de TAS TOTAL siendo el punto de corte mayor o igual a 61 para indicar la presencia de alexitimia. Para nuestro estudio se ha obtenido un índice de fiabilidad alfa de Cronbach .72.

Para valorar la resiliencia se utilizó la Escala de Resiliencia CD-RISC elaborada por Connor y Davidson (2003), traducida al español por Bobes et al. (2001) (citado en Serrano-Parra et al., 2012) y validada en personas mayores de 60 años por Serrano-Parra et al. 2012. Consta de 17 ítems tipo Likert con los siguientes rangos: 0 nunca, 1 casi nunca, 2 a veces, 3 casi siempre y 4 siempre. La escala se compone de tres dimensiones: Tenacidad - Autoeficacia; Control personal y Competencia social. Ha mostrado poseer buenas propiedades psicométricas con un índice alfa de Cronbach de .89 para el conjunto de la escala (Connor y Davidson, 2003) y para cada una de las dimensiones (Serrano-Parra et al., 2012): .79 para Tenacidad - Autoeficacia y Control personal y .56 para Competencia social. Para nuestro estudio el alfa de Cronbach fue de .77 .

Finalmente, se utilizó la Escala de creencias paranormales (Tobacyk, 2004). La escala está formada por 26 ítems que oscilan en los siguientes rangos: 0 muy en desacuerdo; 1 En desacuerdo; 2 De acuerdo; 3 Muy de acuerdo. El instrumento consta de 7 dimensiones: creencias religiosas tradicionales, creencias en la parapsicología, brujería, superstición, espiritualismo, formas de vida extraordinarias y precognición. Para la versión revisada se mejoró la confiabilidad temporal así como la consistencia interna de la prueba (Tobacyk, 2004). El índice de fiabilidad alfa de Cronbach para este estudio fue de .69 .

\section{Procedimiento}

En un primer momento se llevó a cabo una entrevista con el directivo de la institución para poder explicitar los objetivos del estudio, los instrumentos de evaluación y solicitar su autorización. La selección de los sujetos fue a conveniencia atendiendo a los criterios de inclusión mencionados puesto que era necesario que no presentasen un nivel de deterioro global compatible con demencia moderadamente grave o grave según 
la Global Deterioration Scale -Escala de deterioro global- de Reisberg et al. (1982) y que impidiese contestar a los instrumentos de evaluación. A lo largo del estudio se aseguró la confidencialidad y el anonimato de los datos recogidos. La recogida de datos fue llevada a cabo por los autores del estudio desde diciembre del año 2014 hasta febrero del año 2015 .

\section{Análisis de datos}

Para el tratamiento estadístico de los datos se utilizó el programa estadístico informático SPSS, en su versión 19. Para el análisis de los datos descriptivos, se utilizó el estudio de frecuencias, medias, desviaciones típicas y porcentajes. Para el análisis de las variables cuantitativas, se utilizó el coeficiente de correlación de Pearson atendiendo a su significación y al tamaño del efecto.

\section{RESULTADOS}

\section{Alexitimia y Resiliencia}

Cabe mencionar que del total de los sujetos del estudio, 12 presentaban un patrón de tipo alexitímico al tener una puntuación total superior al punto de corte.

En lo que respecta a la alexitimia, cabe destacar que aparecieron correlaciones significativas positivas entre la Dificultad para identificar sentimientos y la Dificultad para describir sentimientos, no siendo así entre la Dificultad para identificar sentimientos y el Patrón de pensamiento orientado a lo externo. Del mismo modo, no hubo correlaciones significativas entre la Dificultad para describir sentimientos y el Patrón de pensamiento orientado a lo externo. Finalmente, aparecieron correlaciones significativas positivas entre el TAS TOTAL y las subescalas de alexitimia (véase tabla 1).

Tabla 1. Correlación de Pearson entre las subescalas de Alexitimia

\begin{tabular}{lcccc}
\hline & 1 & 2 & 3 & 4 \\
\hline 1. Dificultad para identificar sentimientos & 1 & $.498^{* *}$ & .113 & $.779 * * *$ \\
\hline 2. Dificultad para describir sentimientos & $.498 * * *$ & 1 & .295 & $.803 * * *$ \\
\hline 3. Patrón de pensamiento orientado a lo externo & .113 & .295 & 1 & $.607 * * *$ \\
\hline 4. TAS TOTAL & $.779 * * *$ & $.803 * * *$ & $.607 * * *$ & 1 \\
\hline Nota: ***Significativo al nivel .001; **Significativo al nivel .01; *Significativo al nivel .05.
\end{tabular}

En cuanto a la Resiliencia, la correlación de Pearson mostró correlaciones significativas positivas entre: Tenacidad y Autoeficacia y Control personal; Tenacidad y Autoeficacia y Competencia Social así como entre Control personal con Competencia social (véase tabla 2). 
Tabla 2. Correlación de Pearson entre las dimensiones de Resiliencia

\begin{tabular}{lccc}
\hline & 1 & 2 & 3 \\
\hline 1. Tenacidad y autoeficacia & 1 & $.523^{* *}$ & $.440^{* *}$ \\
\hline 2. Control personal & $.523^{* *}$ & 1 & $.377^{*}$ \\
\hline 3. Competencia social & $.440^{* *}$ & $.377^{*}$ & 1 \\
\hline $\begin{array}{l}\text { Nota: ***Significativo al nivel } \\
\text { *Significativo al nivel .05 }\end{array}$ & & & \\
**Significativo al nivel .01 \\
\end{tabular}

Los resultados mostraron que existía una correlación significativa negativa, con tamaño del efecto alto $(r=-.500 ; p=.002 ; N=35)$ entre el Control Personal y la Dificultad para identificar sentimientos (véase gráfico 2). Del mismo modo, existió una correlación significativa negativa, con tamaño del efecto medio entre Control personal y TAS TOTAL $(r=-.374 ; p=.027 ; N=35)$.

Gráfico 2. Gráfico de dispersión para la Dificultad para identificar sentimientos y Control personal

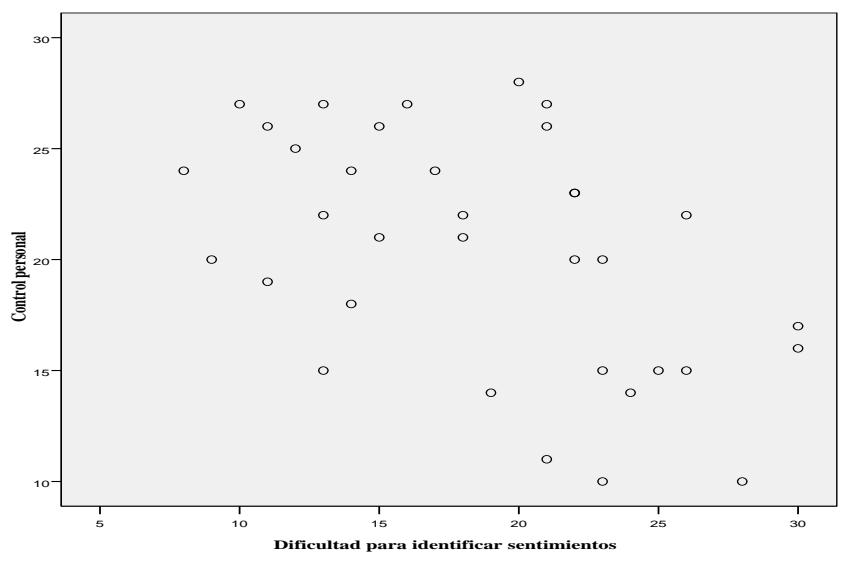

\section{Alexitimia, Resiliencia y creencias paranormales}

En primer lugar en lo que respecta a las creencias paranormales, cabe destacar que se encontraron las siguientes correlaciones de Pearson significativas: Creencias religiosas tradicionales y Espiritualidad con tamaño del efecto medio $(r=.461 ; p=.005$; $N=35)$; Creencia en la parapsicología y Brujería con tamaño del efecto alto $(r=.571$; $p=.000 ; N=35)$; Creencia en la parapsicología y Espiritualidad con tamaño del efecto alto $(r=.578 ; p=.000 ; N=35)$; Creencia en la parapsicología y Precognición con tamaño del efecto alto $(r=.505 ; p=.002 ; N=35)$; Brujería y Creencia en la parapsicología con tamaño del efecto alto $(r=.571 ; p=.000 ; N=35)$; Brujería y Precognición con tamaño del efecto alto $(r=.543 ; p=.001 ; N=35)$;Espiritualidad con Precognición con tamaño del efecto medio $(r=.423 ; p=.011 ; N=35)$; Formas de vida extraordinarias con Precognición con tamaño del efecto medio $(r=.346 ; p=.042 ; N=35)$. 
En segundo lugar, si atendemos a la Alexitimia y las creencias paranormales, los resultados mostraron que existía una correlación negativa media entre la Dificultad para identificar sentimientos y las Formas de vida extraordinarias $(r=-.335 ; p=.049$; $N=35$ ) tal y como puede visualizarse en el gráfico 3.

Gráfico 3 Gráfico de dispersión para la Dificultad para identificar sentimientos y la creencia en Formas de vida extraordinarias

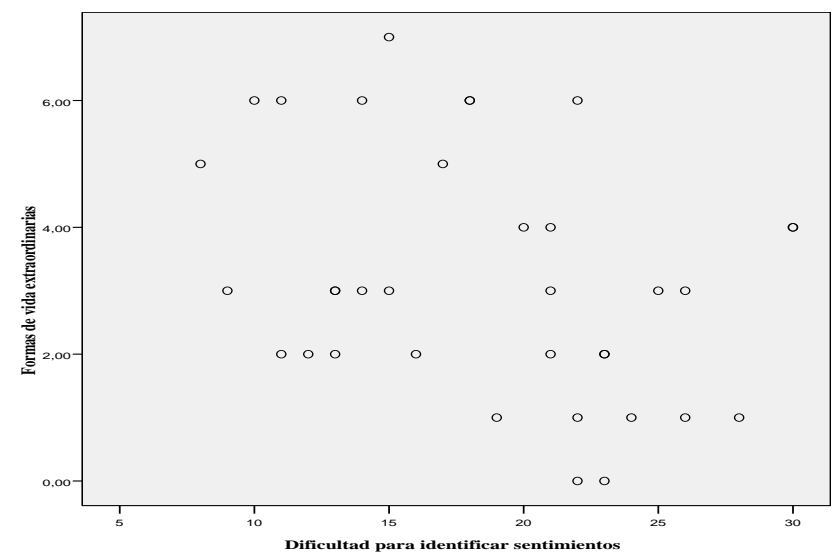

Gráfico 4. Gráfico de dispersión para el Control personal y la creencia en Formas de vida extraordinarias

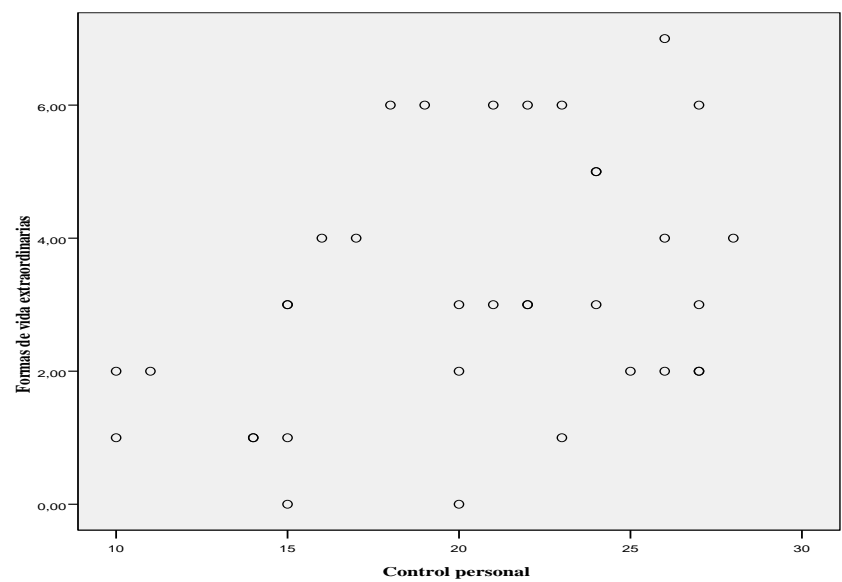

En tercer lugar, en cuanto a la resiliencia, el Control Personal mostró una correlación positiva media con la variable Formas de vida extraordinarias. ( $r=.380$; $p=.024 ; N=35)$, tal y como puede apreciarse en el gráfico 4 . 


\section{DISCUSIÓN}

El estudio ha permitido analizar la Alexitimia, Resiliencia y Creencias paranormales en los ancianos de un centro institucionalizado de Murcia. Es destacable la presencia de 12 sujetos que presentaban las características propias de un patrón de tipo alexitímico. Eso supone que dichos sujetos presentaban las características definitorias de dicho patrón puesto que aparecieron correlaciones significativas entre las subescalas: Dificultad para identificar sentimientos y la Dificultad para describir sentimientos y entre las subescalas de alexitimia y el TAS TOTAL. Estos resultados suponen que los ancianos del estudio que presentaban alexitimia,poseían las características definitorias del mismo (Nemiah, 1977; Martínez-Sánchez et al., 2003; Sivak y Wiater, 1997).

De otro lado, los ancianos mostraron en cuanto a la Resiliencia las características propias del constructo, puesto que se encontraron correlaciones significativas entre las dimensiones de la escala. Estos datos están en la línea con lo apuntado por González-Arratia y Valdez (2013) sobre la importancia de la combinación o interacción dinámica entre los factores internos y externos del sujeto con la finalidad de superar los efectos de los eventos adversos.

En lo que respecta a la Resiliencia y la Alexitimia, comprobamos en el estudio la presencia de una correlación significativa negativa entre el Control Personal y la Dificultad para identificar sentimientos asi como entre Control personal y TAS TOTAL. Esto indica que aquellos sujetos con mayores dificultades para identificar sentimientos son los que presentaban un menor control personal. Estos resultados nos remiten a investigaciones previas que exponían que la alexitimia alude a la dificultad para poder distinguirlos de otras sensaciones corporales, la falta de capacidad para la introspección y que pueden caer en el conformismo social (Nemiah, 1977; Martínez-Sánchez et al., 2003; Sivak y Wiater, 1997).

$\mathrm{Si}$ atendemos a las creencias paranormales, conviene destacar que aparecieron correlaciones significativas entre Creencias religiosas tradicionales y Espiritualidad; Creencia en la parapsicología y Brujería; Creencia en la parapsicología y Espiritualidad; Creencia en la parapsicología y Precognición; Brujería y Creencia en la parapsicología; Brujería y Precognición; Espiritualidad con Precognición; Formas de vida extraordinarias con Precognición.

En lo relativo a la Alexitimia y las creencias paranormales, los resultados mostraron una correlación negativa entre la Dificultad para identificar sentimientos y la creencia en Formas de vida extraordinarias. Es decir, aquellos sujetos con mayores Dificultades para identificar sentimientos son los que menor creencia poseían de las Formas de vida extraordinaria.

Finalmente, en cuanto a la Resiliencia, apareció la existencia de una correlación positiva entre Control Personal y la creencia en Formas de vida 
extraordinarias. Esto es indicativo de que aquellos sujetos con mayor capacidad de Control Personal son los que mayor creencia tenían de las Formas de vida extraordinaria. Estos resultados están en la línea con las investigaciones previas que aluden a que a veces para sobreponerse a situaciones adversas, como puede ser el proceso de institucionalización, hay personas que se apoyan en la religiosidad (Cárdenas y López, 2010), lo cual incluso hace que disminuya la sintomatología ansiosa y depresiva ante la muerte (Parra y Corbetta, 2013) ya que promueve mecanismos psicológicos adaptativos ante el proceso de envejecimiento, la enfermedad y el sufrimiento (Reyes-Ortiz, 1998).

Del mismo modo, se ha comprobado que poseer un sistema de creencias sobre experiencias paranormales se ha asociado a una reducción del nivel de depresión, ansiedad y anhedonia (Parra y Corbetta, 2013). Los datos de nuestro estudio no coinciden con lo apuntado por Irwin (1995) puesto que no encontró relaciones entre Alexitimia y creencias paranormales.

Como aplicabilidad del estudio, cabría mencionar que sería adecuado que los profesionales de centros institucionalizados de personas mayores explorarán las creencias religiosas para poder utilizarlas como recurso complementario en el tratamiento de los ancianos, promoviendo un estilo de vida saludable, dotando de esperanza y sentido de trascendencia, apoyo socioemocional, comunicación, etc. asi como las creencias paranormales como mecanismo de reducción de sintomatología ansiosa o depresiva (Parra y Corbetta, 2013). Asimismo, se recomienda implantar actividades encaminadas a la expresión, reconocimiento y manejo de las emociones a través de programas que promuevan incluso la inteligencia emocional (Pérez-Fuentes, Molero, Gázquez, y Soler, 2014) sobre todo en aquellos sujetos con un patrón de tipo alexitímico.

Como limitaciones es destacable el hecho de que el estudio se ha llevado a cabo con un número limitado de sujetos por lo cual sería adecuado aumentar el tamaño muestral e incluso añadir variables que proporcionen información sobre la calidad de vida (Molero, Pérez-Fuentes, Gázquez, y Mercader, 2012) u otras características personales tales como depresión, ansiedad, etc.

\section{REFERENCIAS}

Bodni, O. (1991). Psicopatología general. Buenos Aires: Editorial Psicoteca

Lolas F. (1978). Event-related slow brain potentials, cognitive processes, and alextithymia. Psychother Psychosom, 30, 116-129. doi:10.1159/000287290

Bagby, R.M., Parker, J.D., y Taylor, G.J. (1994). The twenty-item Toronto Alexithymia Scale. I. Item selection and cross-validation of the factor structure. Journal of Psychosomatic Research, 38, 23-32.

Cárdenas, A., y López, A.L. (2010). Analysis Matrix of Resilience in the Face of Disability, Old Age and Poverty, International Journal of Disability Development and Education, 57(2), 175-189. 
Cárdenas-Jiménez, A., y López-Díaz, A.L. (2011). Resiliencia en la vejez. Revista Salud Pública, 13(3), 528-540.

Connor, K.M., y Davidson, J.R.T. (2003). Development of a new resilience scale: The ConnorDavidson resilience scale (CD-RISC). Depression and Anxiety, 18(2), 76-82.

García-Sevilla, J. Méndez, I., Martínez, J.P., Cánovas, A.B, Clemente, Y., y Boti, Mª́.Á. (2014). Alexitimia, deterioro cognitivo y depresión en personas mayores. En J. J. Gázquez, Ma . C. Pérez Fuentes, Mª . M Molero, I. Mercader y Soler, F. (Eds.), Investigación en Salud y Envejecimiento. Vol 1 (pp.185-212). Almería: Asoc. Univ. de Educación y Psicología (ASUNIVEP) Recuperado de: http://formacionasunivep.com/congresosalud/documents /libro_digital_completo.pdf

Gázquez, J.J., Pérez-Fuentes, M.C., Mercader, I., y Molero, M.M. (2011). Prevalencia de la dependencia funcional en personas mayores. Anales de Psicología, 27(3), 871-876.

González-Arratia, N.I., y Valdez, J.L. (2013). Resiliencia: Diferencias por Edad en Hombres y Mujeres Mexicanos. Acta de Investigación Psicológica, 3(1), 941-955.

Irwin, H. (1995). Las creencias paranormales y las funciones emocionales. Revista Argentina de Psicología Paranormal, 2, 69-76.

Martínez-Sánchez, F. (1996). Adaptación española de la escala de Alexitimia de Toronto (TAS20). Clínica y Salud, 7(1), 19-32.

Martínez-Sánchez, F., Ato-García, M., y Ortiz-Soria, B. (2003). Alexithymia: State or trait? The Spanish Journal of Psychology, 6(1), 51-59. doi: 10.1017/S1138741600005205

Molero, M.M., Pérez-Fuentes, M.C., Gázquez, J.J., y Mercader, I. (2012). Construcción y validación inicial de un cuestionario para evaluar la Calidad de Vida en mayores institucionalizados. European Journal of Investigation in Health, Psychology and Education, 2(2), 53-65. doi: 10.1989/ejihpe.v2i2.14

Nemiah, J.C. (1977). Alexithymia: Theoretical considerations. Psychotherapy and Psychosomatics, 28, 199-206.

Parra, A., y Corbetta, J.M. (2013). Experiencias paranormales y su relación con el sentido de la vida. Liberabit, 19(2), 251-258.

Pérez-Fuentes, M.C., Molero, M.M., y Gázquez, J.J. (2011). Dependencia y valoración de los Servicios Sociales por los mayores. En M.C. Pérez-Fuentes y J.J. Gázquez (Comps.), Envejecimiento y demencia. Un enfoque multidisciplinar (pp.405-414). Granada: GEU Editorial.

Pérez-Fuentes, M.C., Molero, M.M., Gázquez, J.J., y Soler, F.J. (2014). Estimulación de la Inteligencia Emocional en mayores: El programa PECI-PM. European Journal of Investigation in Health, Psychology and Education, 4(3), 329-339. doi: 10.1989/ejihpe .$v 4 i 3.80$

Reisberg, B., Ferris, S., de León, M., y Crook, T. (1982). The Global Deterioration Scale (GDS) for the assessment of primary degenerative dementia. American Journal of Psychiatry, 139(9), 1136-1139. doi: 10.1176/ajp.139.9.1136

Reyes-Ortiz, C.A. (1998). Importancia de la religión en los ancianos. Colombia Médica, 29(4), 155-157.

Serrano-Parra, M.D., Garrido-Abejar, M., Notario-Pacheco, B., Bartolomé-Gutierrez, R., Solera-Martínez, M., y Martínez-Vizcaíno, V. (2012). Validez de la escala de Resiliencia de Connor-Davidson (CD-RISC) en una población de mayores entre 60 y 75 años. International Journal of Psychological Research, 5(2), 49-57. Recuperado de: http://mvint.usbmed.edu.co:8002/ojs/index.php/web/index

Sivak, R., y Wiater, A. (1997). Alexitimia, la dificultad para verbalizar afectos. Teoría y Clínica. Buenos Aires, Argentina: Editorial Paidós SAICF. 
Tobacyk, J. (2004). A Revised Paranormal Belief Scale. The International Journal of Transpersonal Studies, 23, 94-98.

Recibido: 4 de junio de 2015 Recepción Modificaciones: 31 de julio de 2015 Aceptado: 4 de agosto de 2015 